\title{
CLUMPS IN THE PLANETARY NEBULAE
}

\author{
A.F. KHOLTYGIN \\ S.-Petersburg Univ. Astron. Obs., 198904, Petergof, Russia \\ and \\ T.KH. FEKLISTOVA \\ Tartu Astrophysical Observatory, EE2444, Tõravere, Estonia
}

\begin{abstract}
The effect of density and temperature fluctuations on the intensity of the recombination and collisionally excited lines is investigated.

The abundances of CNO ions in planetary nebulae found from the recombination lines often exceed those from collisionally excited lines. We have investigated the dependance of the ratio $\left\{X_{\text {rec }}\right\} /\left\{X_{\text {coll }}\right\}$ versus $\left\{X_{\text {coll }}\right\}$ where $\left\{X_{\text {rec }}\right\}$ and $\left\{X_{\text {coll }}\right\}$ are respectively the abundancies of the ion $X$ determined from the recombination and collisional excited lines. The negative correlation between the values $\left\{X_{\text {rec }}\right\} /\left\{X_{\text {coll }}\right\}$ and $\left\{X_{\text {coll }}\right\}$ for planetaty nebulae type II is found. It is proposed to use the ratio of the abundances determined using the lines dominated by different line formation mechanisms as the measure of the electron temperature and ion density fluctuations in planetary nebulae. The effect of the fluctuations on the intensities of the recombination lines and collisionally excited lines are investigated. Dimensions of dense clumps in the planetary nebulae are estimated. We have proposed that the clumps are the relic of the maser condensations in the $\mathrm{OH} / \mathrm{IR}$ progenitors. Discrepancy in the $\mathrm{C}, \mathrm{N}$ and $\mathrm{O}$ abundances determined from recombination lines and collisionally excited lines is a real fact. This discrepancy is due to the thermal and density inhomogenities in planetary nebulae.
\end{abstract}

